\title{
Soft Materials that Intercept, Respond to, and Sequester Bacterial Siderophores
}

Benjamin J. Ortiz, ${ }^{1}$ James Jennings, ${ }^{2}$ William S. Gross, ${ }^{3}$ Thiago M. A. Santos, ${ }^{3}$ Ti-Yu Lin, ${ }^{3}$ Douglas B. Weibel, ${ }^{2,3,4, *}$ and David M. Lynn ${ }^{1,2, *}$

${ }^{1}$ Department of Chemical and Biological Engineering, 1415 Engineering Dr.; ${ }^{2}$ Department of Chemistry, 1101 University Ave.; ${ }^{3}$ Department of Biochemistry, 433 Babcock Dr.; ${ }^{4}$ Department of Biomedical Engineering, 1550 Engineering. Dr.; University of Wisconsin-Madison, Madison, WI 53706; "Email: (D.B.W.) douglas.b.weibel@gmail.com; (D.M.L.) dlynn@engr.wisc.edu

\section{Supporting Information}

SI Table 1. Bacterial strains used in this study.

\begin{tabular}{|c|c|c|}
\hline Bacterial strain & Genotype or description & Source or reference \\
\hline E. coli BW25113 & $\begin{array}{l}F \text {-, } \Delta(\text { araD-araB }) 567, \Delta \text { lacZ4787(::rrnB- } \\
\text { 3), } \lambda^{-} \text {rph-1, } \Delta(\text { rhaD-rhaB) 568, hsdR514 }\end{array}$ & $\begin{array}{l}\text { Laboratory collection, }{ }^{1} \\
\text { CGSC\#7636 }\end{array}$ \\
\hline E. coli BW25113 & BW25113, sentC $731::$ kan (E. coli & Keio collection, ${ }^{2}$ CGSC\# \\
\hline$\Delta e n t C$ & JW0585) & 8702 \\
\hline E. coli BW25113 & BW25113, $\Delta$ fur-731::kan (E. coli & Keio collection, ${ }^{2}$ \\
\hline$\Delta f u r$ & JW0669) & CGSC\#8758 \\
\hline
\end{tabular}

${ }^{1}$ Datsenko, K. A., Wanner, B. L. One-step inactivation of chromosomal genes in Escherichia coli K-12 using PCR products. Proc. Natl. Acad. Sci., U. S. A. 2000, 97, 6640-6645.

${ }^{2}$ Baba, T. et al. Construction of Escherichia coli K-12 in-frame, single-gene knockout mutants: the Keio collection. Mol. Syst. Biol. 2006, 2, 2006.0008.

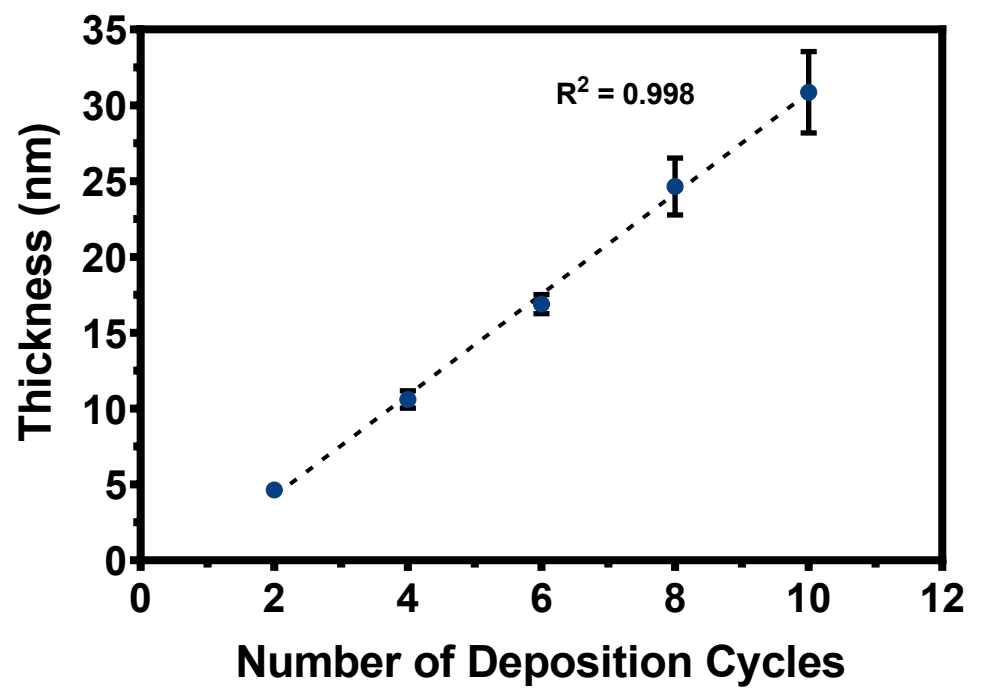

Figure S1. Plot showing optical thickness as a function of the number of TA-Fe deposition cycles on a silicon wafer. The data points represent the average of three independent samples for which thickness was measured at five different surface positions. 


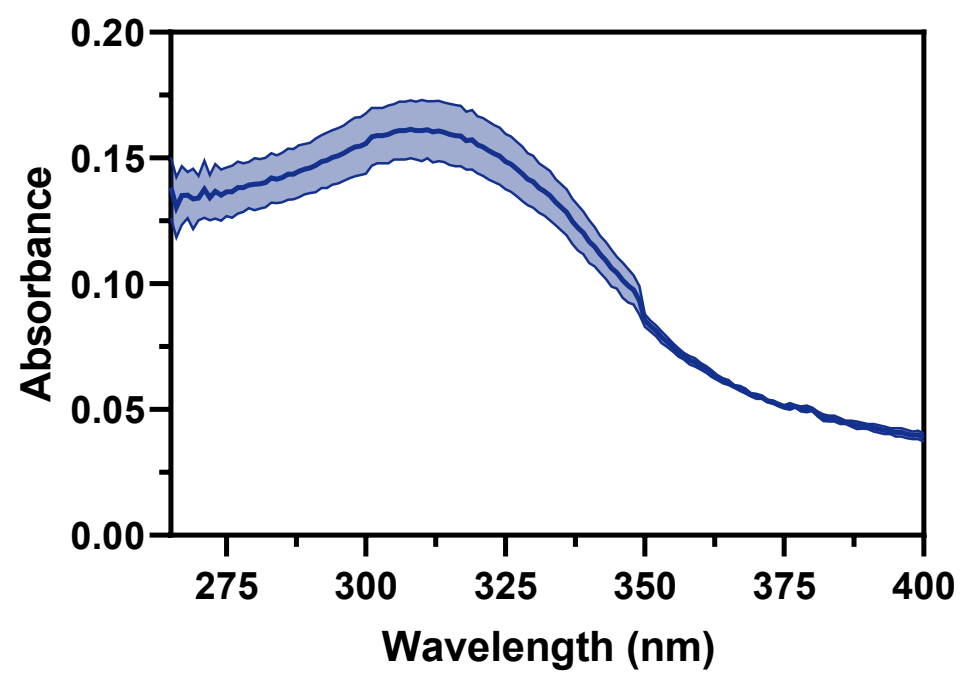

Figure S2: UV/vis absorption spectra for (TA-Fe) ${ }_{10}$ coatings deposited on glass slides. The blue line represents the average absorbance value of three independent coatings after 10 deposition cycles and the SEM is represented by outlining blue shadow.
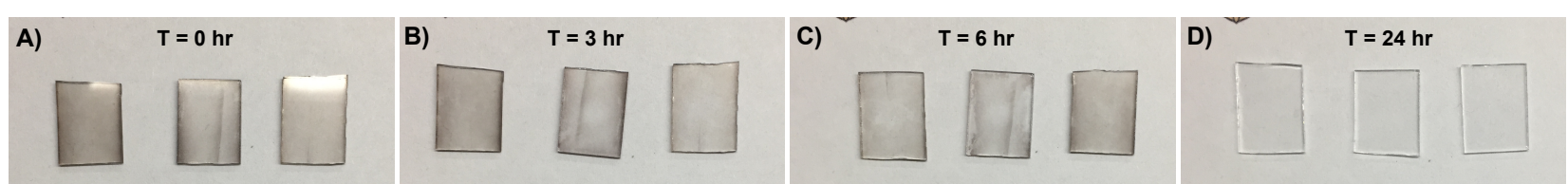

Figure S3. Photographs of three independently coated (TA- $\left.\mathrm{Fe}^{\mathrm{III}}\right)_{10}$ glass slides with $1.3 \mathrm{~cm}$ by $1.5 \mathrm{~cm}$ dimensions (A) immediately after fabrication, and (B-D) after incubation with $4 \mu \mathrm{M}$ concentrations of enterobactin at $37^{\circ} \mathrm{C}$ for (B) 3 hours, (C) 6 hours, and (D) 24 hours. 


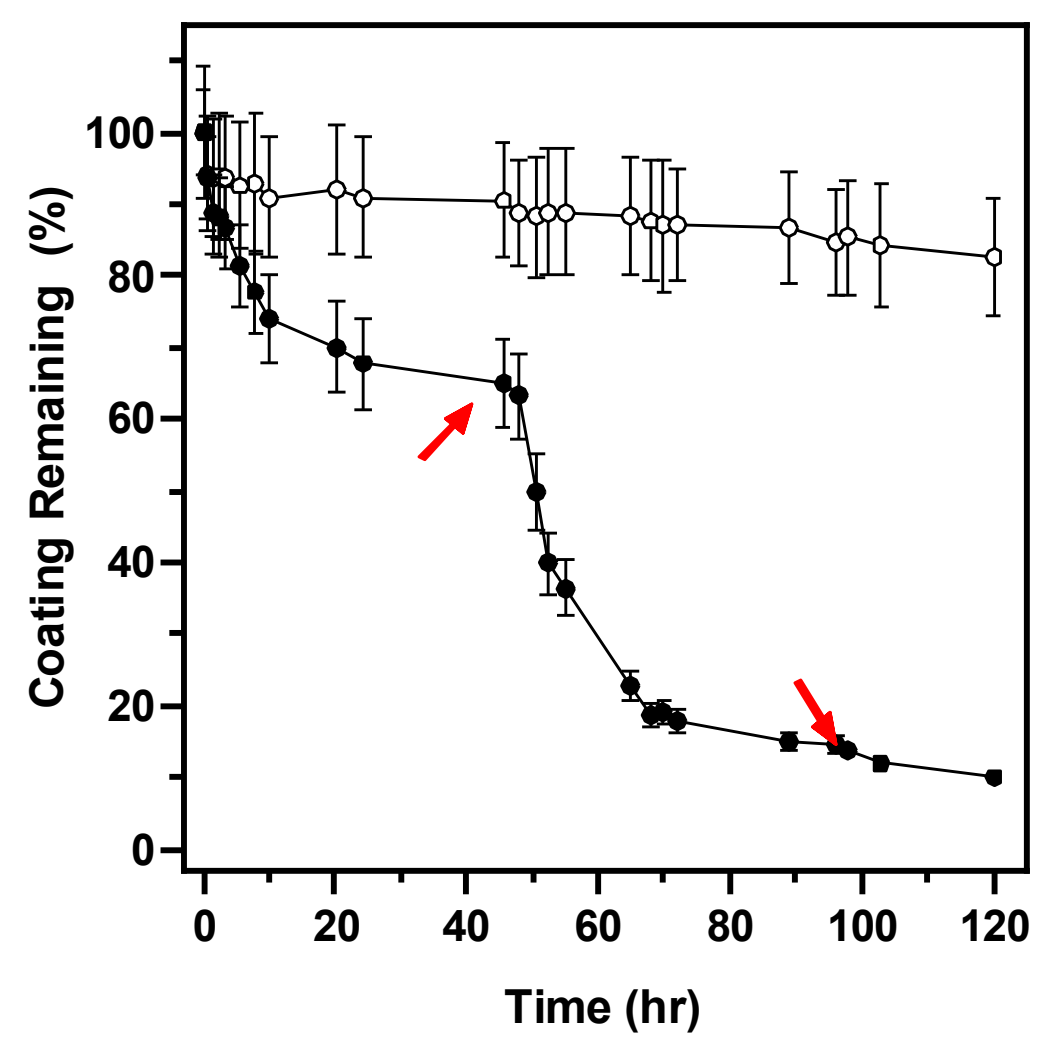

Figure S4. Plot showing degradation of TA-Fe ${ }^{\mathrm{III}}$ films coated on glass slides upon incubation in $2 \mu \mathrm{M}$ enterobactin in 9:1 water/acetonitrile (black circles) and in 9:1 water/acetonitrile (open circles) solutions at $37^{\circ} \mathrm{C}$. The enterobactin solution was replenished every 48 hours (at time points indicated by red arrows). The error bars represent standard error. 


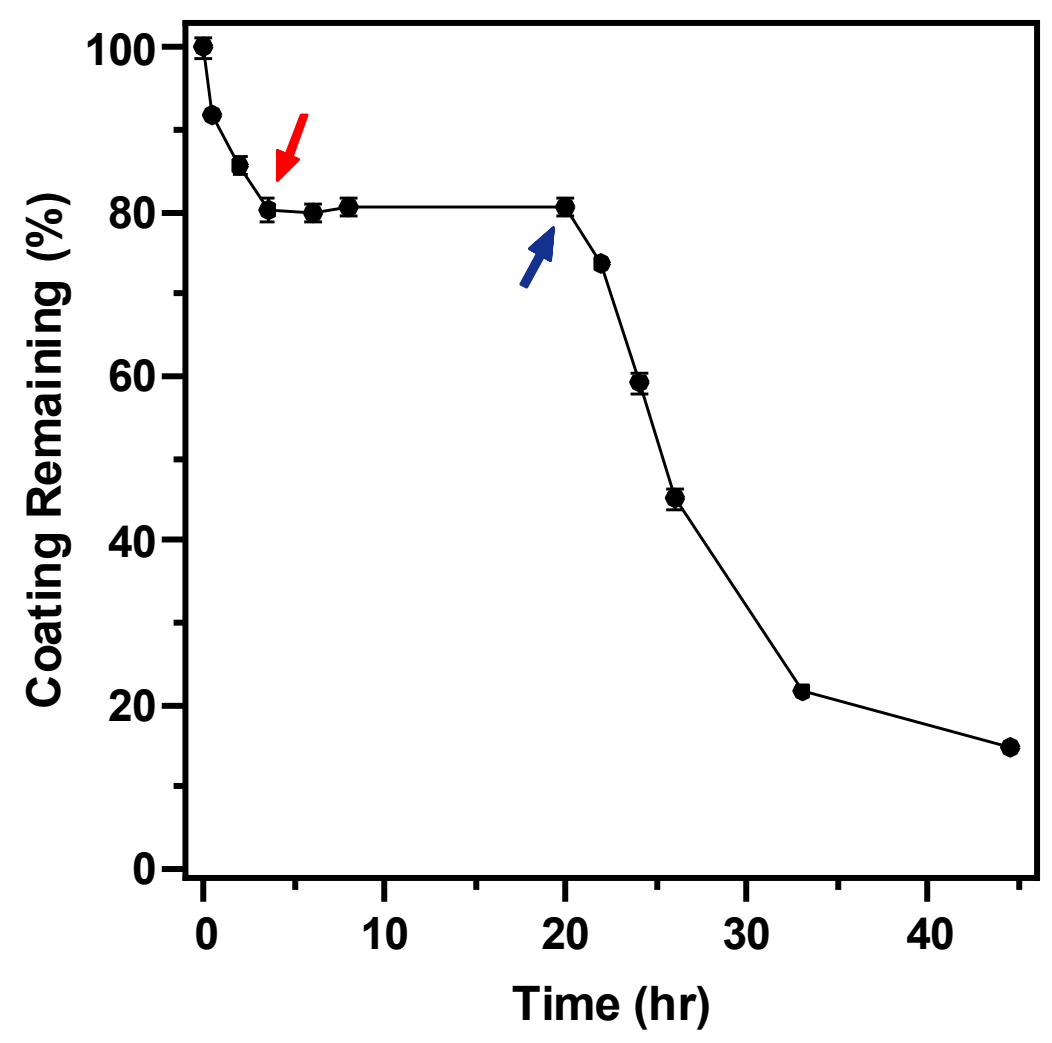

Figure S5. Plot showing degradation of $\mathrm{TA}-\mathrm{Fe}^{\mathrm{III}}$ films coated on glass slides in $4 \mu \mathrm{M}$ enterobactin in 9:1 water/acetonitrile solutions. The enterobactin was replaced with 9:1 water/acetonitrile solution at the time point denoted by the red arrow, upon which degradation was arrested. After replacement of medium with $4 \mu \mathrm{M}$ enterobactin (indicated by the blue arrow), degradation resumed. Error bars denote standard error. 


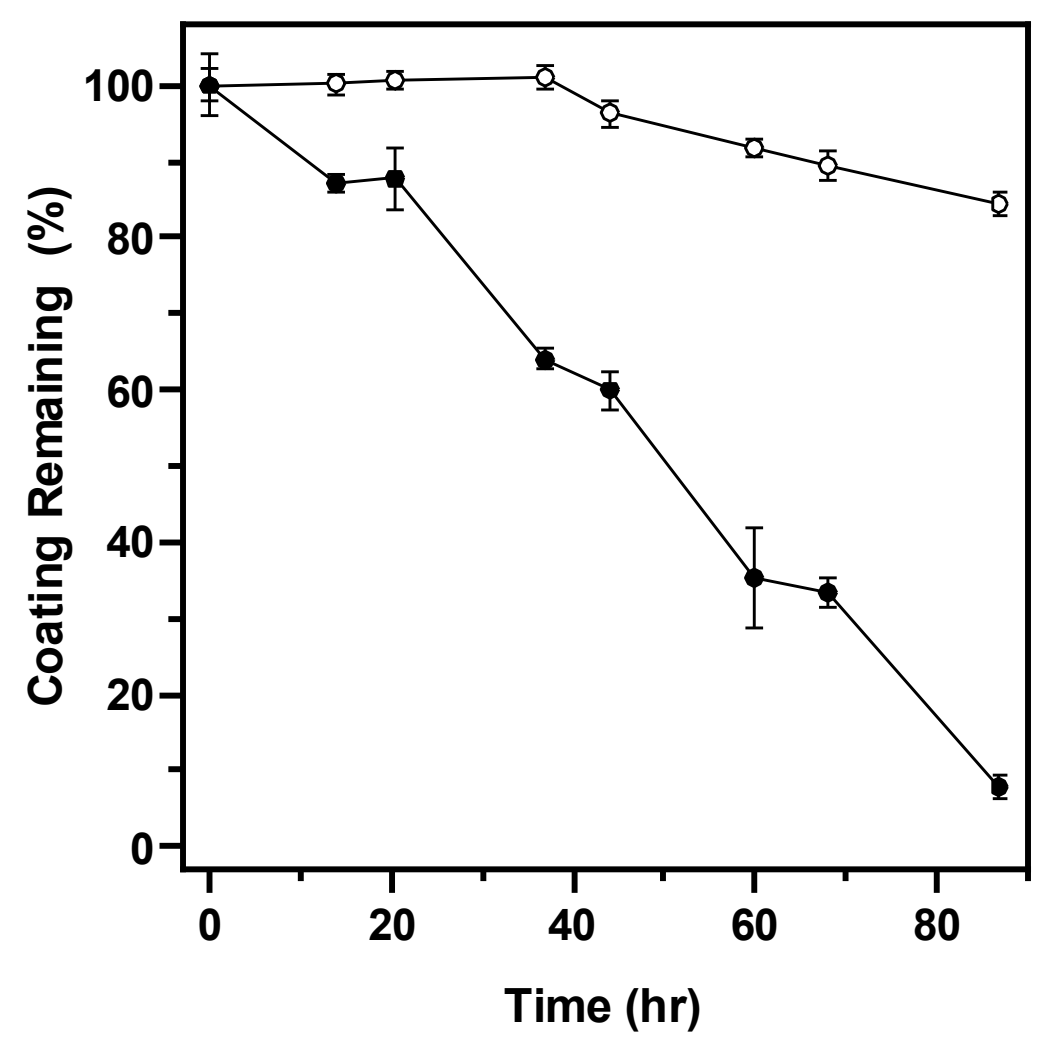

Figure S6. Plot showing degradation of TA-Fe ${ }^{\mathrm{III}}$ films coated on glass slides in the presence of $200 \mu \mathrm{M}$ DFO in PBS (filled circles) and in PBS only (open circles). Error bars denote standard error. 


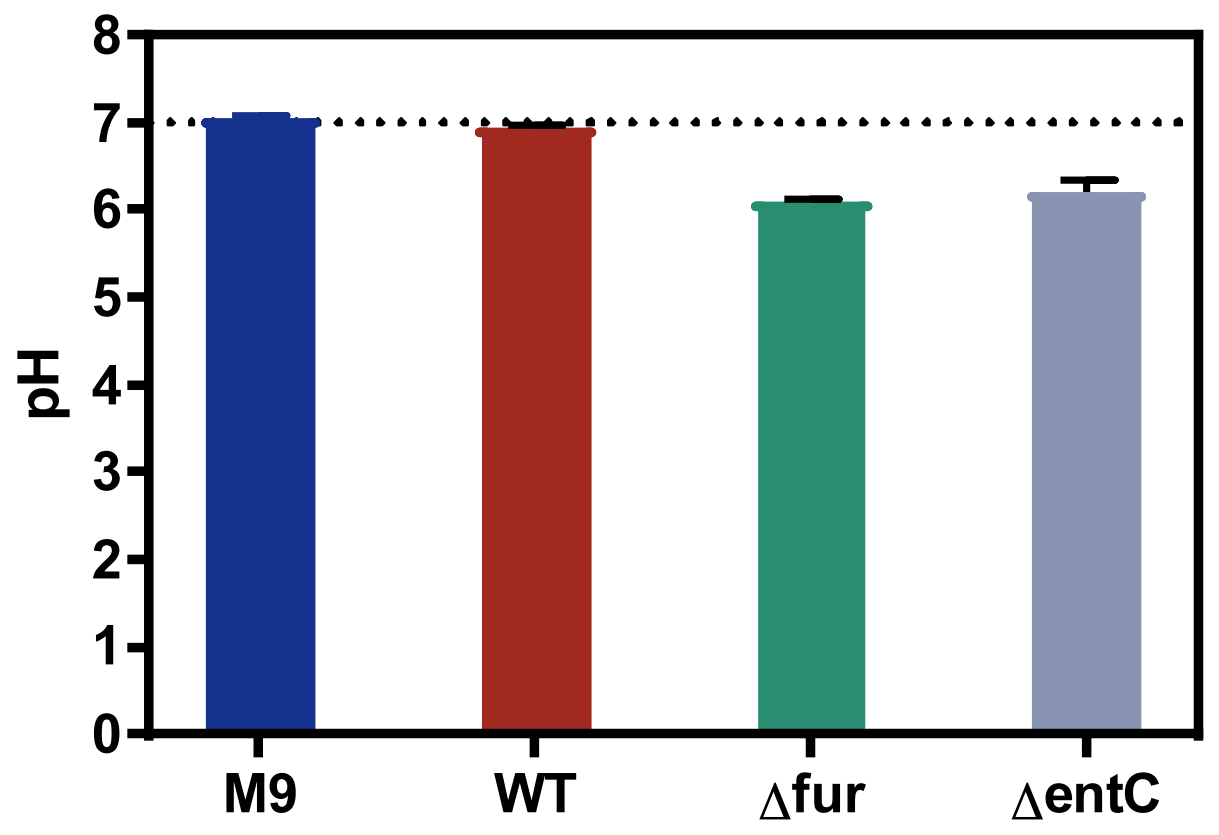

Figure S7. Evaluation of the $\mathrm{pH}$ of culture supernatants for different $E$. coli strains after 48 hours of growth in M9 media at $37{ }^{\circ} \mathrm{C}$. E. coli it is known to metabolically acidify its local environment by, for example, producing acetic acid, ${ }^{62}$ and these changes in $\mathrm{pH}$ could also potentially lead to the disassembly of TA-Fe coatings. Therefore, the $\mathrm{pH}$ of $E$. coli culture supernatants was measured to rule out $\mathrm{pH}$ as a possible erosion mechanism. Each bar represents the mean values obtained from three different replicates with error bars representing the standard error of the mean. 


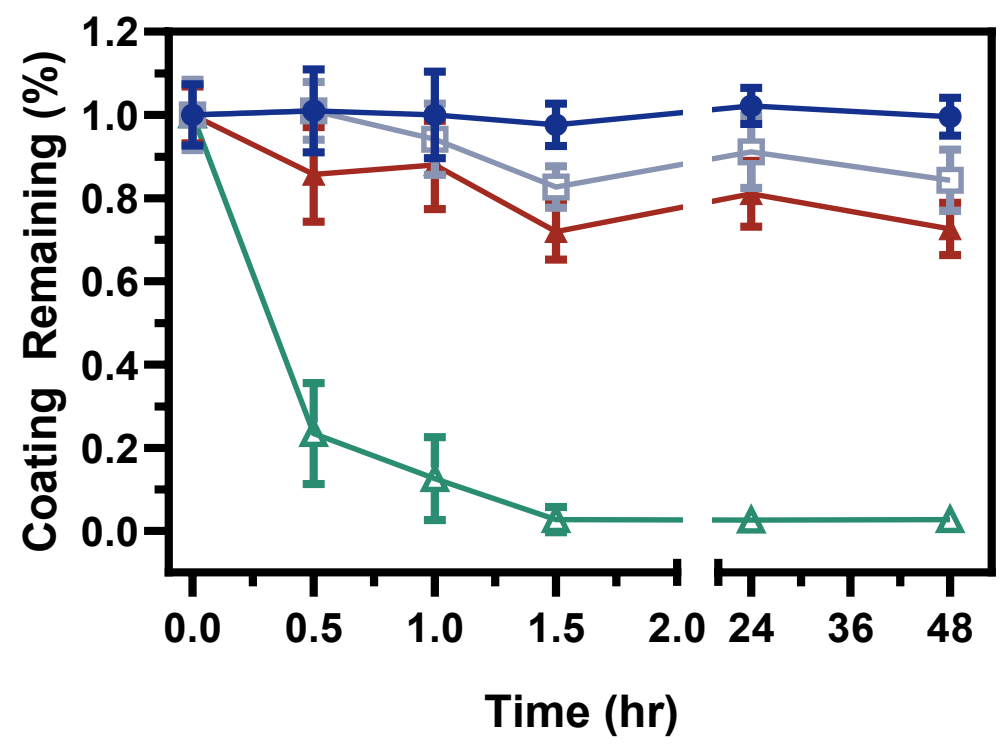

Figure S8. Evaluation of the stability of (TA-Fe) $)_{10}$ over time upon incubation in M9 minimal media supplemented with amino acids and $\mathrm{pH}$-adjusted with $\mathrm{HCl}$ to 7 (blue filled circles), 5 (gray open squares), 4 (brown filled triangles) and 2 (green open triangles) at $37{ }^{\circ} \mathrm{C}$. The coatings remain stable down to a $\mathrm{pH}$ of 4 in $\mathrm{M} 9$ media at which the TA-Fe $\mathrm{III}^{\mathrm{III}}$ mono-complex predominates. ${ }^{20}$ The percentage of coating remaining represents the absorbance of coatings at $315 \mathrm{~nm}$ normalized with respect to the initial absorbance of coatings measured at time $=0$. All data points represent the mean $\pm \operatorname{SEM}(n=3)$.

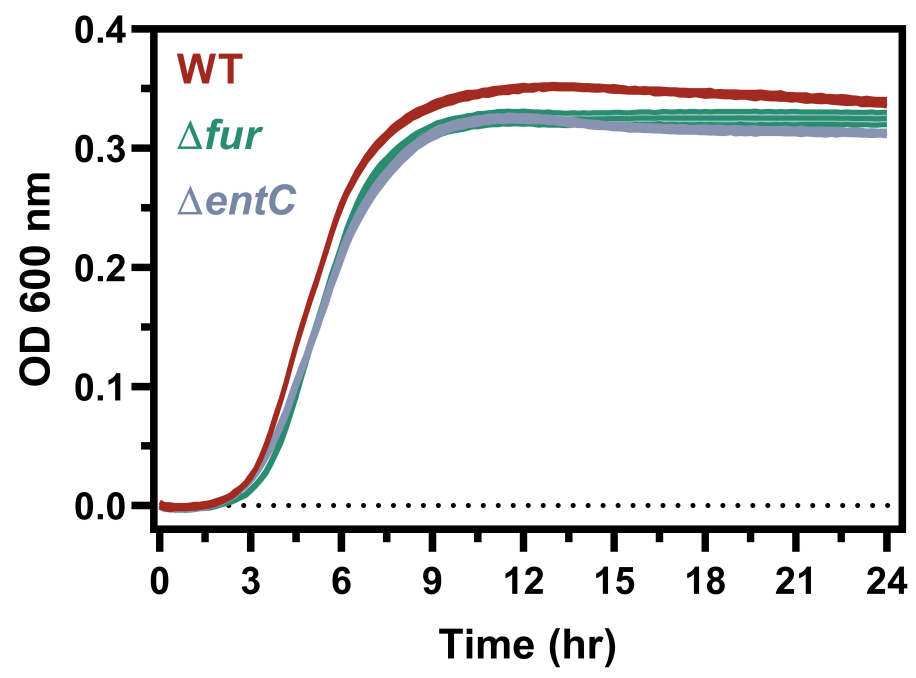

Figure S9. Evaluation of the growth of E. coli strains measured by following optical density (OD) over time as a measure of absorbance at $\lambda=600 \mathrm{~nm}$. The strains were grown in M9 media supplemented with amino acids under orbital shaking at $37^{\circ} \mathrm{C}$. The OD curves shown are an average with standard error of three independent experimental replicates. 


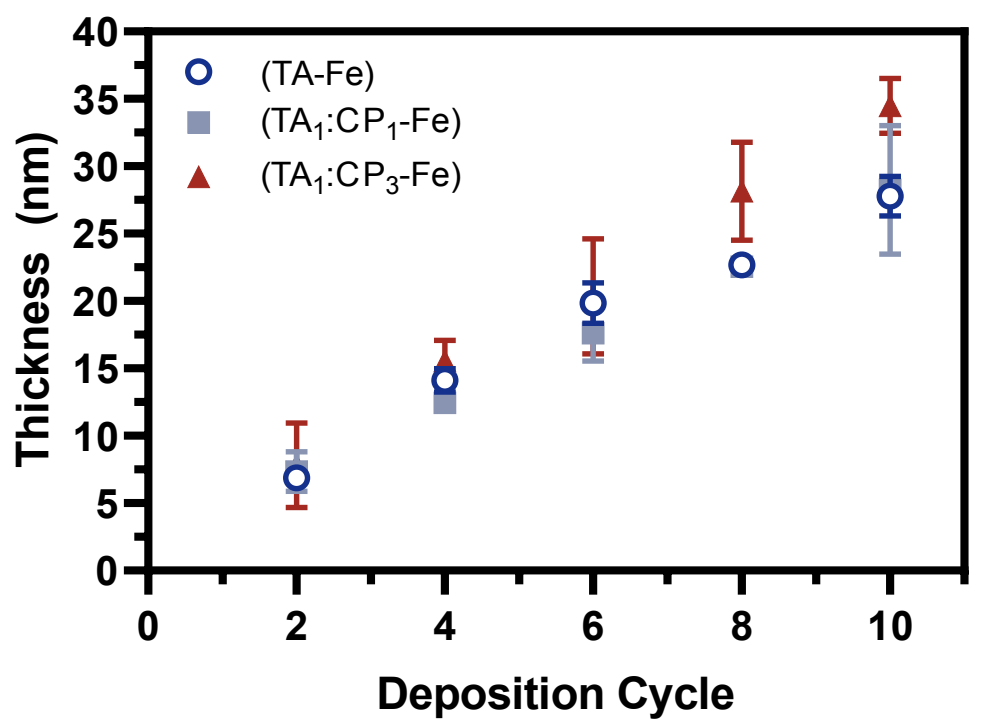

Figure S10. Optical thickness as a function of the number of deposition cycles of TA-Fe (open circles), $\mathrm{TA}_{1}: \mathrm{CP}_{1}-\mathrm{Fe}$ (filled squares), and $\mathrm{TA}_{1}: \mathrm{CP}_{3}-\mathrm{Fe}$ (filled triangles) coatings on a silicon wafer. The optical thickness represents the average of three independent samples for which thickness was measured at five different surface positions.

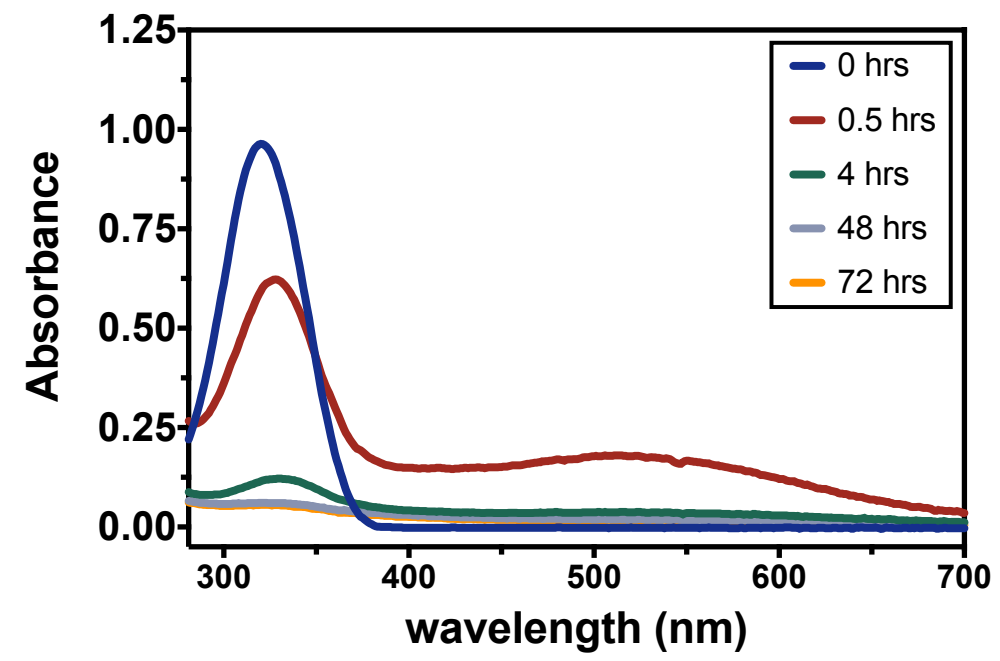

Figure S11. UV/vis absorption spectra of the supernatant of a $100 \mu \mathrm{M}$ enterobactin solution in water that was incubated with $\mathrm{Fe}$-alginate beads at $37^{\circ} \mathrm{C}$ for the indicated amounts of time. 


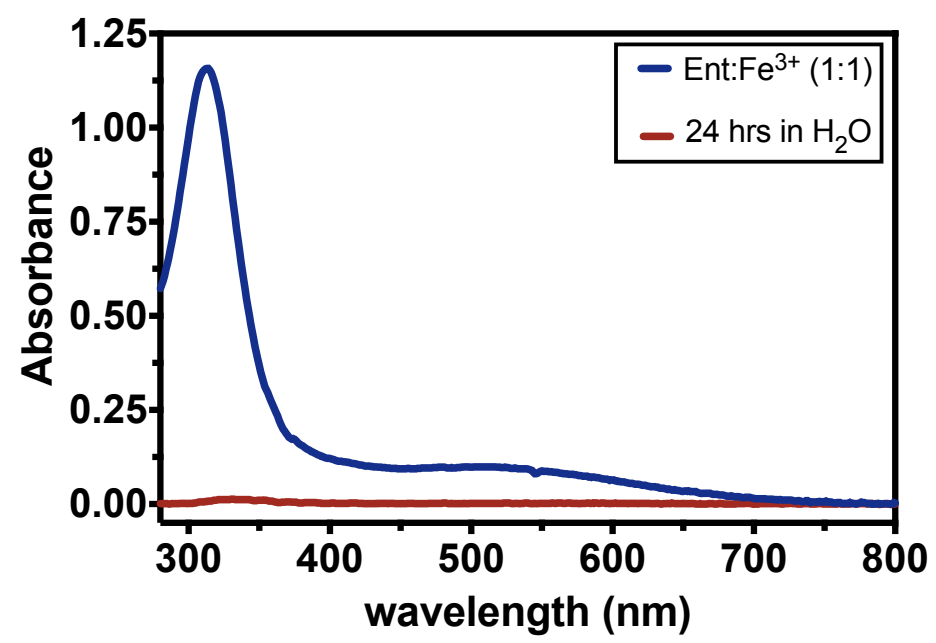

Figure S12. UV/vis spectra of a solution consisting of a 1:1 molar mixture of enterobactin and $\mathrm{Fe}^{\mathrm{III}}$ (blue) and spectra of aqueous supernatant that was incubated for 24 hours at $37^{\circ} \mathrm{C}$ with $\mathrm{Fe}-$ alginate beads containing sequestered enterobactin (red).

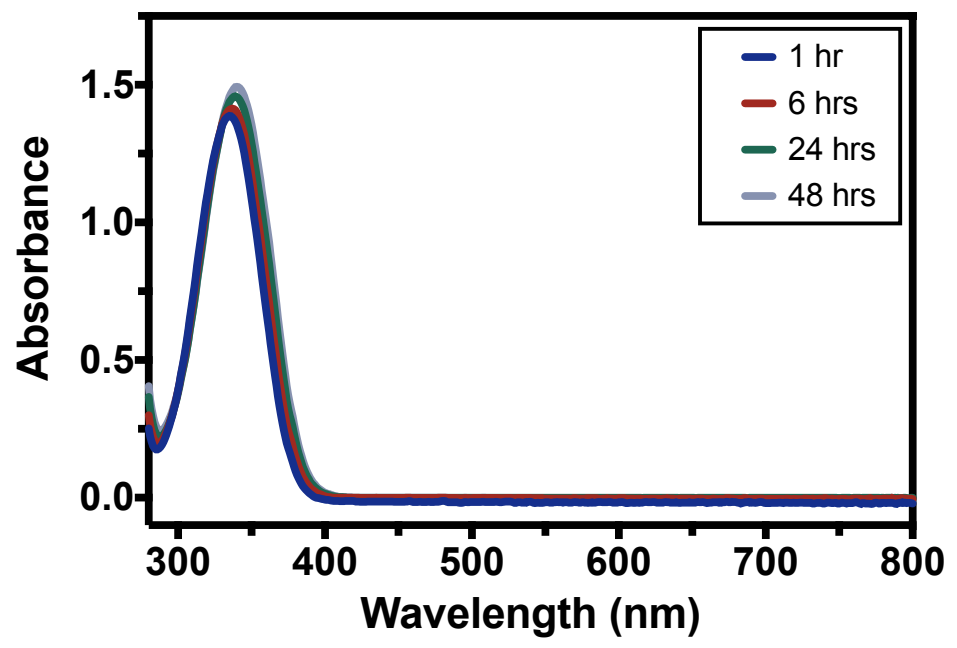

Figure S13. UV/vis absorption spectra of the supernatant of a $100 \mu \mathrm{M}$ enterobactin solution in water that was incubated with Ga-alginate beads at $37^{\circ} \mathrm{C}$ for the indicated amounts of time. 


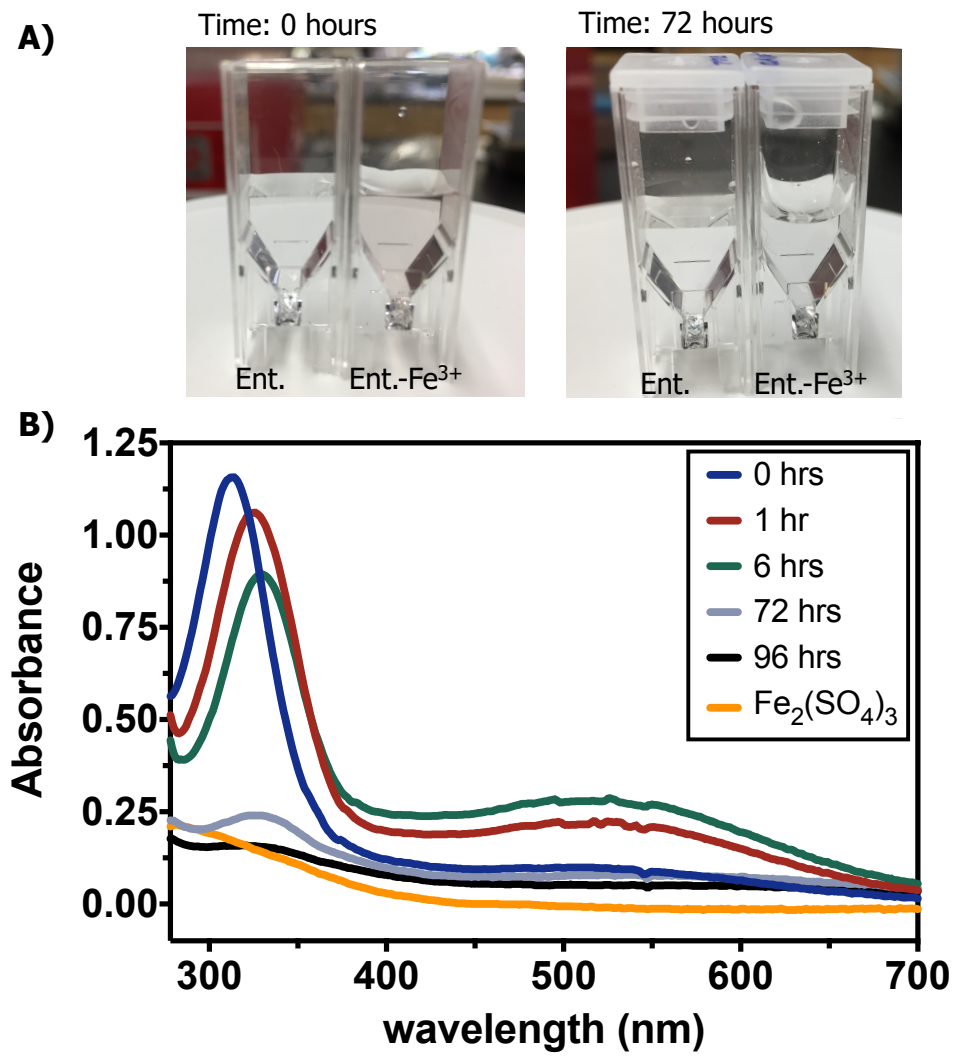

Figure S14. (A) Photographs of enterobactin solutions and enterobactin/ $/ e^{\mathrm{III}}$ equimolar mixtures before incubation with Fe-alginate beads (left) and after 72 hours of incubation with Fe-alginate beads at $37^{\circ} \mathrm{C}$. (B) UV/vis spectra of enterobactin/Fe $/$ III equimolar mixtures after incubation with $\mathrm{Fe}$-alginate beads for predetermined periods at $37^{\circ} \mathrm{C}$ in water. 